\title{
Biceps tendon rupture diagnosed by physical examination and ultrasonography in the emergency department
}

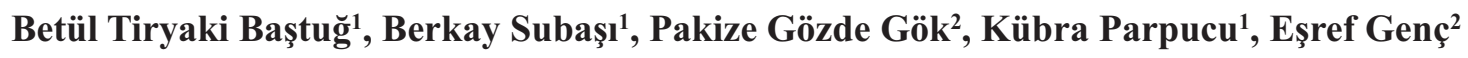 \\ ${ }^{1}$ Department of Radiology, Osmangazi University School of Medicine, Eskişehir, Turkey \\ ${ }^{2}$ Department of Emergency Medicine, Osmangazi University School of Medicine, Eskişehir, Turkey
}

\begin{abstract}
A biceps brachii rupture can occur at either superior or inferior end but most commonly involves the long head at its proximal attachment to superior glenoid labrum. We report a 48 -year-old male with a proximal long-head biceps rupture because of trauma diagnosed by ultrasonography and physical examination in the emergency department. On physical examination, there was a severe pain over the anterior aspect of the shoulder, proximal part of the biceps muscle, and distally located biceps muscle mass. In ultrasonographical study, there were no tendon fibers in the right shoulder bicipital groove. The evaluation of both the physical examination and sonographic findings revealed a proximal long-head biceps rupture.
\end{abstract}

Eur Res J 2018;4(3):235-237

Keywords: Biceps tendon, rupture, ultrasound

\section{Introduction}

A biceps brachii rupture can occur at either superior or inferior end but most commonly involves the long head at its proximal attachment to superior glenoid labrum $[1,2]$. Trauma-related injury involves the distal part, which is rare and seen in young people [3]. Degenerative causes involve the proximal part of the tendon. We report a case with a proximal longhead biceps rupture because of trauma diagnosed by ultrasonography and physical examination in the emergency department.

\section{Case Report}

A 48-year-old male was admitted to our emergency department with sudden onset of right arm pain. He reported that he was lifting a heavy piece of machinery when he heard a sudden popping sound and now was complaining of significant swelling to his right arm.

The patient was previously healthy. He has smoking for 30 pack per year. On physical examination, there was a severe pain and tenderness over the anterior aspect of the shoulder, proximal part of the biceps muscle, and distally located biceps muscle mass (Figure 1). The right elbow active and passive range of motion was full, but there was a mild weakness with 4 of 5 powers in manual testing during elbow flexion and supination. Neurovascular examination and conventional radiographic imaging 


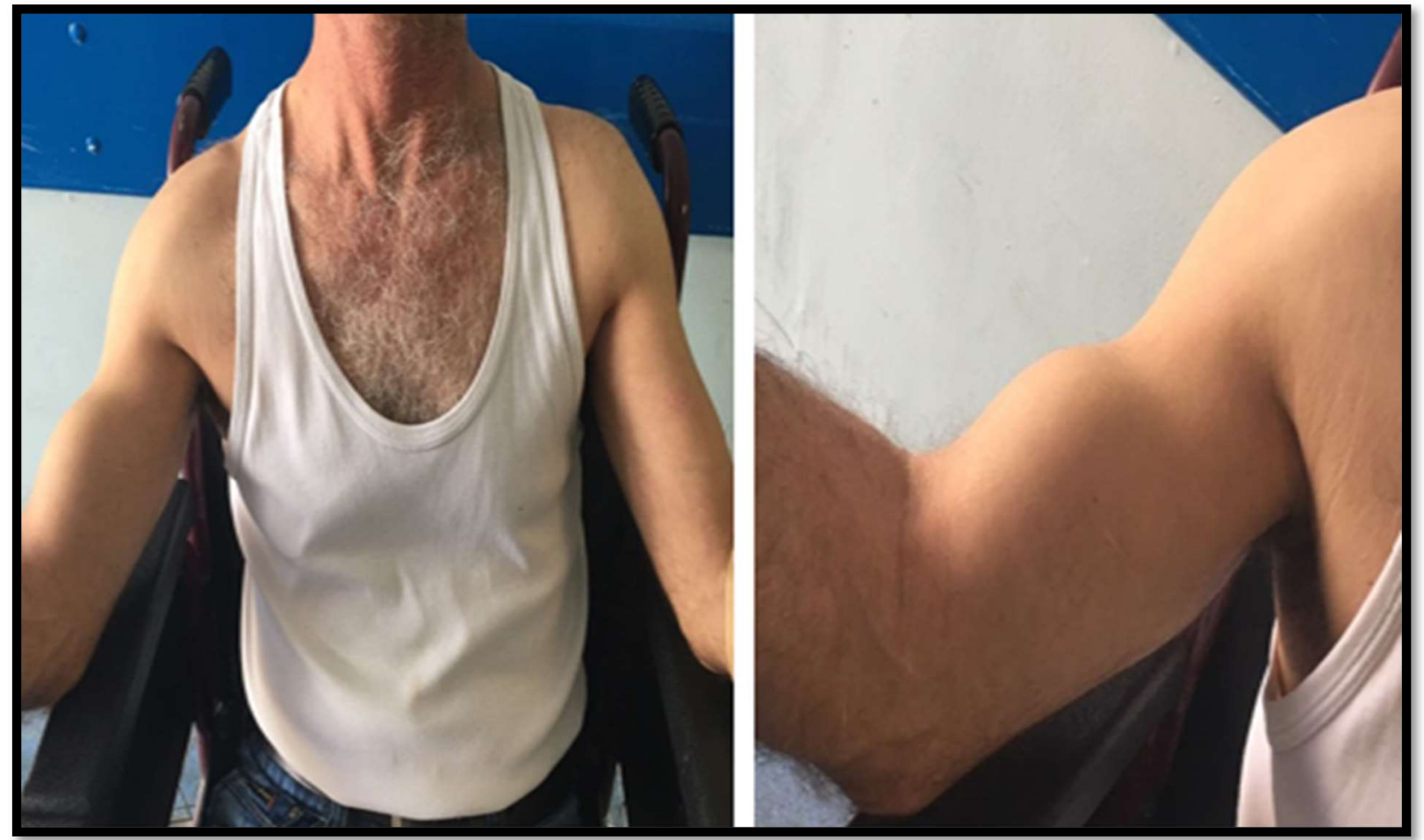

Figure 1. On physical examination, there was a severe pain and tenderness over the anterior aspect of the shoulder, proximal part of the biceps muscle, and distally located biceps muscle mass.

findings were normal. An emergency radiologist performed ultrasound (LOGIQ P5; GE Medical System, China) with a 7.5- to $12-\mathrm{MHz}$ linear transducer. Ultrasound was performed while the patient was sitting, and his hand was positioned palm upward on the knee. The linear transducer was placed on the right bicipital area, and longitudinal-transverse scans were obtained. In ultrasound, there were no tendon fibers in the right shoulder bicipital groove
(Figure 2). The evaluation of both the physical examination and sonographic findings revealed a proximal long-head biceps rupture. The patient was discharged from emergency department with a conservative treatment. He was placed in a sling and given pain medicine. Elective magnetic resonance imaging (MRI) was planned. He followed up in the orthopedic clinic three days later where the decision was made for surgical repair given his occupation as

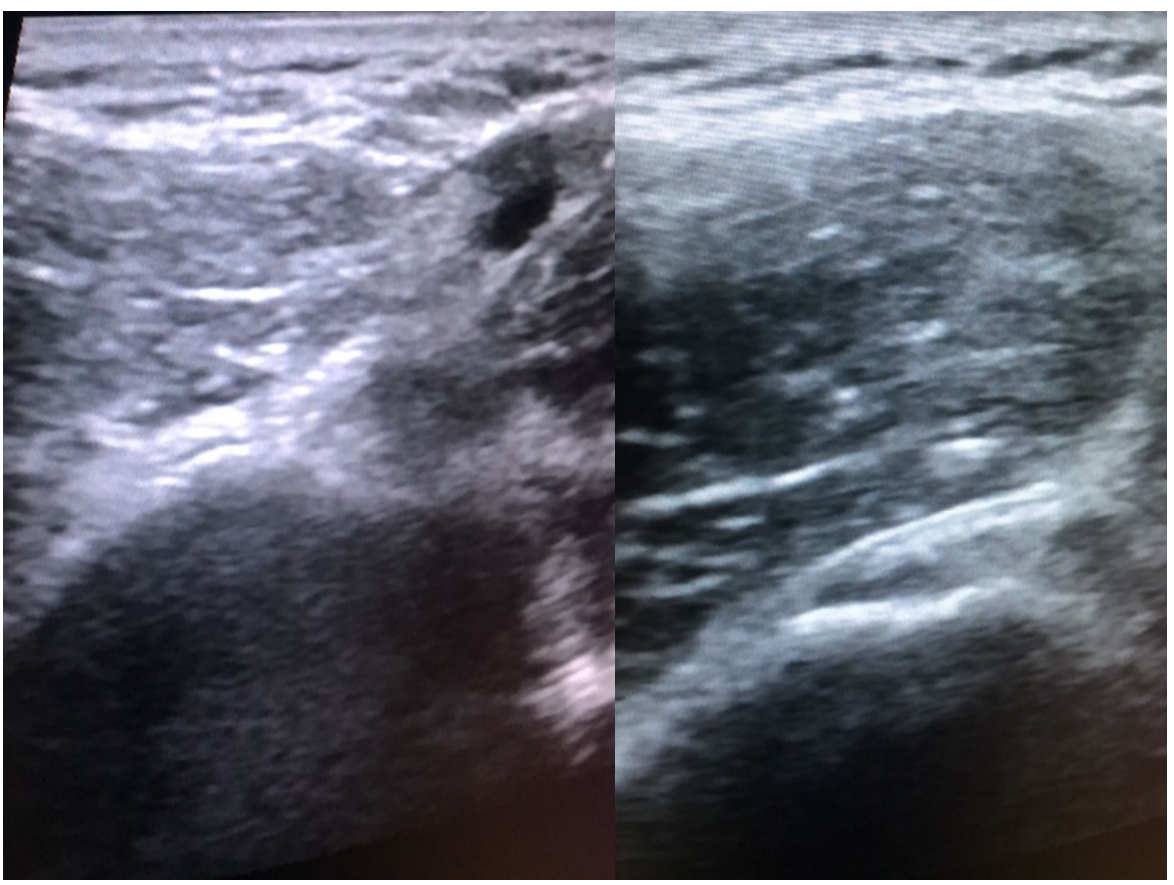

Figure 2. In ultrasound, there were no tendon fibers in the right shoulder bicipital groove 
a mechanic recommendation after orthopedic evaluation.

\section{Discussion}

The biceps brachii muscle is employed in supination and flexion of the forearm. The biceps muscle has two proximal tendon additions on the scapula - the long head and the short head. The long head originates from the supraglenoid tubercle and extends over the humeral head into the intertubercular groove of the humerus. The short head arises from the coracoid process along with the coracobrachialis muscle medial to the long head tendon. The distal tendon of the biceps muscle joins to the radial tuberosity [1].

The great majority of biceps tendon ruptures occur at the proximal insertion and almost always involve the long head [1]. Most often, these ruptures occur at the bony attachment or the tendon-labral junction. Distal tendon ruptures are uncommon but can occur at the insertion on the radial tuberosity. Tendon ruptures also can happen at the short head insertion on the acromion, although this is less common.

Persons aged 40-60 years who have a history of shoulder problems that cause chronic strain on the tendon are at the highest risk for biceps tendon rupture [2]. Traumatic ruptures that occur in younger ones are usually the result of acute strain on the tendon, such as heavy weight-lifting or a traumatic fall. These often are the result of the forced extension of the elbow. Other risk factors for tendon ruptures include chronic disease states such as diabetes, chronic kidney disease, systemic lupus erythematosus, rheumatoid arthritis, chronic steroid use, fluoroquinolone use, and cigarette smoking [3].

The diagnosis of these injuries is usually made clinically [4]. Patients typically apply after an acute traumatic event where the patient experienced a sudden onset of pain, heard a "pop," and noticed bruising or swelling. Many patients with biceps tendon rupture also have a history of chronic shoulder pain due to nerve impingement.

Physical examination involves inspection of bilateral upper extremities; a biceps tendon rupture often presents as a visible or palpable mass and is referred to as a "Popeye" deformity. An examination of the affected arm should be done, and any tenderness along the biceps tendon and muscle belly should be noted.

Plain radiography is generally not diagnostic. With these graphies you can rule out bony injuries.
Ultrasound is defined as a reliable indicator of biceps tendon rupture $[5,6]$. MRI is often considered the "gold standard" and able to demonstrate the anatomy of the biceps tendon rupture, but is usually not applied in the emergency department.

There is debate about the final treatment of biceps tendon ruptures. Emergency care should focus on comfort measures, such as muscle relaxation by using analgesia, anti-inflammatories, and a hanger. Prompt orthopedic or sports medicine follow up is warranted for these patients and non-emergent imaging can be done for evaluation for possible surgical management. Some biceps tendon ruptures, especially those involving the long head, can be managed conservatively with pain control and physical therapy [7].

\section{Conclusion}

The diagnosis of biceps brachii tendon rupture can be easily made with physical examination and ultrasonography is a reliable imaging modality in detection of this injury.

\section{Informed consent}

Written informed consent was obtained from the patient for the publication of this case report.

\section{Conflict of interest}

The authors declared that there are no potential conflicts of interest with respect to the research, authorship, and/or publication of this article.

\section{References}

[1] Elser F, Braun S, Dewing CB, Giphart JE, Millett PJ. Anatomy, function, injuries, and treatment of the long head of the biceps brachii tendon. Arthroscopy 2011;27:581-92.

[2] Carter AM, Erickson SM. Proximal biceps tendon rupture: primarily an injury of middle age. Phys Sportsmed 1999;27:95-102.

[3] Marx JA, Hockberger RS, Walls RM. Rosen's Emergency Medicine Concepts and Clinical Practice, 2 Volume Set, 8th edition, Elsevier: Sanders 2014.

[4] O'Driscoll SW, Goncalves LB, Dietz P. The hook test for distal biceps tendon avulsion. Am J Sports Med 2007;35:1865-9.

[5] Skendzel JG, Jacobson JA, Carpenter JE, Miller BS. Long head of biceps brachii tendon evaluation: accuracy of preoperative ultrasound. AJR Am J Roentgenol 2011;197:942-8.

[6] Lobo Lda G, Fessell DP, Miller BS, Kelly A, Lee JY, Brandon C, et al. The role of sonography in differentiating full versus partial distal biceps tendon tears: correlation with surgical findings. AJR Am J Roentgenol 2013;200:158-62.

[7] Mariani EM, Cofield RH, Askew LJ, Li GP, Chao EY. Rupture of the tendon of the long head of the biceps brachii. Surgical versus nonsurgical treatment. Clin Orthop Relat Res 1988;228:233-9. 